\title{
Rituximab purging and maintenance therapy combined with autologous stem cell transplantation in patients with diffuse large B-cell lymphoma
}

\author{
WEI ZHANG, LI JIAO, DAO-BIN ZHOU and TI SHEN \\ Department of Hematology, Peking Union Medical College Hospital, Chinese Academy of \\ Medical Sciences and Peking Union Medical College, Beijing 100730, P.R. China
}

Received February 23, 2010; Accepted April 30, 2010

DOI: 10.3892/ol_00000128

\begin{abstract}
The aim of this prospective, single-arm study was to test the efficacy and tolerability of autologous stem cell transplantation (auto-SCT) combined with in vivo rituximab purging and post-transplant rituximab maintenance therapy in patients with diffuse large B-cell lymphoma (DLBCL). This study included 12 DLBCL patients aged 18-65 years with an International Prognostic Index $\geq 2$. The patients received 4-6 cycles of induction therapy consisting of rituximab plus cyclophosphamide, adriamycin, vincristine and prednisone followed by salvage therapy prior to stem cell mobilization. This regimen was followed by rituximab maintenance therapy $\left(375 \mathrm{mg} / \mathrm{m}^{2}\right.$ every three months for two years). Prior to auto-SCT, six patients $(50 \%)$ achieved complete remission (CR) and six $(50 \%)$ achieved unconfirmed complete remission (CRu). Three months after transplantation, 11 patients (91.7\%) achieved CR and one achieved $\mathrm{CRu}$. After two cycles of rituximab maintenance therapy, all 12 patients achieved CR. Long-term CR was achieved by 10 patients, while two experienced relapse at 14 and 20 months after the end of rituximab maintenance therapy. The median follow-up period was 44 months (range 35-61). Disease-free survival was noted in 10 patients, while two experienced relapse. The three-year overall survival (OS) and progression-free survival (PFS) were 100 and 83\%, respectively. Prolonged hypogammaglobulinemia occurred in two patients, although no increase in major infections was observed. Hepatitis B surface antigen was continuously negative in all 12 patients. Our results demonstrated that auto-SCT combined with in vivo rituximab purging and post-transplant rituximab maintenance is safe and effective, and may extend OS and PFS in younger high-risk DLBCL patients.
\end{abstract}

Correspondence to: Professor Dao-Bin Zhou, Department of Hematology, Peking Union Medical College Hospital, Chinese Academy of Medical Sciences and Peking Union Medical College, Beijing 100730, P.R. China

E-mail: vv1223@vip.sina.com

Key words: diffuse large B-cell lymphoma, autologous stem cell transplantation, rituximab purging, rituximab maintenance

\section{Introduction}

Diffuse large B-cell lymphoma (DLBCL) is the most common form of non-Hodgkin's lymphoma (1). Prognostic factors include age, general health, serum lactate dehydrogenase levels, Ann Arbor disease stage, and the presence of extranodal lesions (2). The introduction of the anti-CD20 antibody rituximab greatly improved the management and treatment of B-cell malignancies (3), including DLBCL $(4,5)$. Retrospective analyses of rituximab in combination with cyclophosphamide, doxorubicin, vincristine, prednisone (CHOP) or equivalent regimens demonstrated that the two-year overall survival (OS) increased to $78 \%$ in adult DLBCL patients of all ages, in comparison to $52 \%$ in the pre-rituximab era (6). For DLBCL patients, combination therapy consisting of rituximab plus CHOP chemotherapy is now the standard first-line treatment (5).

Despite improved outcomes, a proportion of DLBCL patients exhibit relapse, particularly the subgroup of patients with an International Prognostic Index (IPI) $\geq 2$ (according to National Comprehensive Cancer Network criteria). Currently, the standard therapy for chemosensitive relapses is based on salvage chemotherapy followed by high-dose therapy and autologous stem cell transplantation (auto-SCT) in selected patients (7). The benefits of auto-SCT in combination with salvage chemotherapy over salvage chemotherapy alone have been demonstrated in several studies $(8,9)$. In this clinical setting, the application of rituximab for in vivo purging of stem cell grafts resulted in a high purging efficiency and improved response rates $(10,11)$. Five-year interim results from an ongoing study have shown the efficacy of in vivo rituximab graft purging and post-transplant rituximab maintenance therapy in combination with auto-SCT in patients with relapsed follicular lymphoma (12). However, these results need to be confirmed in studies using additional patient cohorts, such as DLBCL patients. Another unknown element is the effect of rituximab induction treatment on the outcome of second-line treatment regimens involving the use of rituximab for graft purging and maintenance therapy. Further clinical evidence is necessary in order to guide the choice of salvage chemotherapy regimens and assess the role of rituximab maintenance following auto-SCT. 
This study aimed to determine the efficacy and safety of rituximab purging and maintenance therapy combined with auto-SCT in younger patients with high-risk DLBCL after rituximab-based induction therapy.

\section{Materials and methods}

Inclusion criteria. DLBCL patients were evaluated at the Department of Hematology, Peking Union Medical College Hospital, China, between December 2004 and December 2006. The main inclusion criteria were: an IPI score $\geq 2$, age 18-65 years, an Eastern Cooperative Oncology Group (ECOG) status <2, and partial recovery after corresponding induction therapy. Patients were excluded if they exhibited central nervous system involvement, a history of other malignancies, pregnancy, severe non-malignant cardiac, renal, hepatic or neurological diseases, and severe active infections, including evidence of hepatitis B virus (HBV) replication. The study protocol was approved by the local ethics committee, and this study was performed in accordance with the standards defined by the 1964 Declaration of Helsinki. Informed consent was obtained from all patients prior to enrollment in the study.

Induction therapy. All 12 patients received 4-6 courses of induction therapy consisting of rituximab plus CHOP. The patients underwent evaluation after every two cycles of chemotherapy. Patients who did not exhibit partial remission (PR) or who exhibited relapse after remission received salvage chemotherapy, which consisted of 2-4 courses of etoposide, cisplatin, cytarabine and methylprednisolone/mitoxantrone, ifosfamide and etoposide until PR was achieved. Stem cells were mobilized in patients without lymphoma infiltration as assessed by bone marrow examination.

Mobilization and harvesting of autologous peripheral hematopoietic stem cells. Peripheral hematopoietic stem cells were mobilized with CHOP (cyclophosphamide: $2.5 \mathrm{~g} / \mathrm{m}^{2}$ ). Granulocyte colony-stimulating factor (G-CSF) was administered at a concentration of $5-10 \mu \mathrm{g} / \mathrm{kg} / \mathrm{day}$. A single infusion of rituximab $(600 \mathrm{mg})$ was administered intravenously as an in vivo purge one day prior to stem cell mobilization. Peripheral hematopoietic stem cells were harvested using a Baxter CS3000 Plus cell separator when peripheral mononuclear cells (MNCs) were $>1.5 \times 10^{9} / 1$ with a target count of $>2 \times 10^{8}$ MNCs and $>2 \times 10^{6} \mathrm{CD} 34^{+}$cells per kg of body weight. Patients in complete (CR) or unconfirmed complete remission $(\mathrm{CRu})$ after mobilization underwent auto-SCT.

Transplantation of hematopoietic stem cells. The patients were pretreated with carmustine $\left(300 \mathrm{mg} / \mathrm{m}^{2}\right.$, day -7$)$, etoposide $\left(200 \mathrm{mg} / \mathrm{m}^{2}\right.$, days -6 to -3$)$, cytarabine $\left(400 \mathrm{mg} / \mathrm{m}^{2}\right.$, days -6 to -3$)$ and melphalan $\left(140 \mathrm{mg} / \mathrm{m}^{2}\right.$, day -2$)$ (BEAM). One day after stem cell reinfusion, G-CSF ( $5 \mu \mathrm{g} / \mathrm{kg} /$ day $)$ was administered subcutaneously until the WBC count reached $>1.5 \times 10^{9} / 1$. Antimicrobial prophylaxis was administered simultaneously.

In vivo purging with rituximab. A single infusion of rituximab $\left(375 \mathrm{mg} / \mathrm{m}^{2}\right)$ was administered intravenously as an in vivo purge one day before stem cell mobilization, one day before conditioning therapy (day -8) and seven days after reinfusion $($ day +7$)$. Dexamethasone and Tylenol ${ }^{\circledR}$ were administered for allergy prevention prior to infusion.

Rituximab maintenance after stem cell transplantation. At 100 days after auto-SCT, patients underwent rituximab $\left(375 \mathrm{mg} / \mathrm{m}^{2}\right)$ maintenance therapy every three months for a total of two years.

Treatment of hepatitis $B$. Patients who were positive for hepatitis B-associated antibodies (with the exception of HBsAb) received lamivudine ( $0.1 \mathrm{mg}$ orally once daily).

Patient follow-up. Follow-up was carried out every three months, and included a physical examination, blood testing, testing for HBV serological markers and quantification of serum immunoglobulins. A whole body computed tomography (CT) scan was performed every six months. Response to therapy was assessed every six months. Patients exhibiting relapse were withdrawn from the study and received salvage treatment. Rituximab treatment was withdrawn when treatment-associated adverse effects were greater than second degree.

Endpoints and statistical considerations. The primary outcome in this study was progression-free survival (PFS). Secondary endpoints were OS and the presence of treatmentrelated toxicity. OS was defined as the interval between stem cell reinfusion and patient death, and PFS was defined as the time between stem cell reinfusion and the first relapse or last follow-up. Treatment efficacy was evaluated according to the standardized response criteria for lymphomas (13). OS and PFS were determined using the Kaplan-Meier method.

\section{Results}

Patient demographics and baseline characteristics. Seven male and five female patients with stage III/IV DLBCL were recruited in the present study. Patient baseline characteristics are summarized in Table I. The mean age was 45 years (range 18-63). One patient had an IPI score of 4 , while the remaining patients exhibited IPI scores of 2-3. The patients received an average of seven cycles of chemotherapy (range 6-9) prior to undergoing auto-SCT. First-line auto-SCT was performed in nine patients. Among these patients, residual disease was found in three individuals without CR. Another three patients who showed chemosensitive disease received salvage treatment due to relapse, and $\mathrm{CRu}$ was achieved after salvage treatment followed by auto-SCT.

Response to therapy and relapse. Prior to undergoing autoSCT, six patients (50\%) had achieved CR and six patients (50\%) had achieved CRu. Three months after transplantation, 11 patients (91.7\%) achieved CR and only one patient achieved $\mathrm{CRu}$. After two cycles of rituximab maintenance therapy, CR was achieved in all 12 patients. A total of 10 patients achieved long-term CR and the other two experienced relapse 14 and 20 months after the end of rituximab maintenance therapy. Of these two patients, one experienced a relapse before transplantation, and CR was achieved after salvage treatment and stem cell mobilization. After four cycles of rituximab maintenance 
Table I. Patient baseline characteristics.

\begin{tabular}{|c|c|c|c|c|c|c|c|c|}
\hline $\begin{array}{l}\text { Patient } \\
\text { no. }\end{array}$ & Gender & $\begin{array}{c}\text { Age } \\
\text { (years) }\end{array}$ & $\begin{array}{l}\text { Ann } \\
\text { Arbor } \\
\text { stage }\end{array}$ & IPI & $\begin{array}{c}\text { No. of } \\
\text { previous } \\
\text { chemotherapy } \\
\text { regimens }\end{array}$ & $\begin{array}{l}\text { Remission } \\
\text { status before } \\
\text { autologous } \\
\text { SCT }\end{array}$ & $\begin{array}{c}\text { Remission } \\
\text { status after } \\
\text { autologous } \\
\text { SCT }\end{array}$ & $\begin{array}{r}\text { Status post- } \\
\text { rituximab } \\
\text { maintenance } \\
\text { (months) }\end{array}$ \\
\hline 1 & M & 36 & III & 2 & 6 & CR1 & $\mathrm{CR}$ & CCR 57+ \\
\hline 2 & $\mathrm{M}$ & 30 & III & 2 & 6 & CR1 & $\mathrm{CR}$ & CCR 55+ \\
\hline 3 & $\mathrm{~F}$ & 28 & IV & 3 & 7 & CRu1 & $\mathrm{CR}$ & CCR 55+ \\
\hline 4 & M & 36 & IV & 3 & 6 & CR1 & $\mathrm{CR}$ & CCR 53+ \\
\hline 5 & M & 18 & III & 2 & 6 & CR1 & $\mathrm{CR}$ & CCR 53+ \\
\hline 6 & $\mathrm{M}$ & 63 & III & 3 & 6 & CRu1 & $\mathrm{CR}$ & CCR45+ \\
\hline 7 & $\mathrm{~F}$ & 45 & IV & 3 & 9 & $\begin{array}{c}\text { Relapse } 1 \\
\text { sensitive } \\
(\mathrm{CRu})\end{array}$ & $\mathrm{CR}$ & CCR41+ \\
\hline 8 & M & 55 & III & 2 & 6 & CRu1 & $\mathrm{CRu}$ & Relapse, CR \\
\hline 9 & M & 47 & IV & 3 & 9 & $\begin{array}{c}\text { Relapse } 1 \\
\text { sensitive } \\
(\mathrm{CRu})\end{array}$ & $\mathrm{CR}$ & Relapse, CR \\
\hline 10 & $\mathrm{~F}$ & 35 & IV & 2 & 6 & CR1 & $\mathrm{CR}$ & CCR $35+$ \\
\hline 11 & $\mathrm{~F}$ & 60 & III & 2 & 8 & $\begin{array}{c}\text { Relapse } 1 \\
\text { sensitive } \\
(\mathrm{CRu})\end{array}$ & $\mathrm{CR}$ & CCR 34+ \\
\hline 12 & $\mathrm{~F}$ & 57 & IV & 4 & 7 & CR1 & $\mathrm{CR}$ & CCR 33+ \\
\hline
\end{tabular}

IPI, International Prognostic Index; SCT, stem-cell transplantation; CR, complete remission; CRu, unconfirmed complete remission; CCR, continuous complete remission.

therapy (14 months after auto-SCT), space-occupying lesions were found, indicating a relapse. The other patient had a residual lesion in the neck before undergoing transplantation. This lesion showed partial improvement after auto-SCT. After two cycles of rituximab maintenance therapy the residual lesion was resolved, suggesting CR. However, a relapse was noted after six cycles of rituximab maintenance therapy (20 months after auto-SCT). Subsequently, the two patients received salvage therapy and radiotherapy, which led to CR.

Survival. The mean follow-up period was 44 months (range 35-61). Disease-free survival was noted in 10 patients, and two patients survived, despite tumor relapse. The median survival time was not achieved in either patient. The threeyear OS rate was $100 \%$ and the three-year PFS rate was $83 \%$. The projected five-year OS and PFS rates are 100 and $83 \%$, respectively (Fig. 1).

Adverse effects. No serious adverse events or deaths related to stem cell mobilization or auto-SCT were noted in this study, nor any treatment-related mortalities. During stem cell harvesting, the median MNC count was $3.8 \times 10^{8}$ per $\mathrm{kg}$ of body weight (range $2.2-7.1 \times 10^{8}$ ) and the median $\mathrm{CD}^{3} 4^{+}$cell count was $4.6 \times 10^{6}$ per $\mathrm{kg}$ of body weight (range $2.5-8.4 \times 10^{6}$ ). The median time for the recovery of neutrophils was 10 days (range 8-11). After auto-SCT, eight patients experienced fever due to agranulocytosis. No symptoms of infection were observed in seven patients. One patient had septicemia caused by $E$. coli but recovered after antibiotic therapy. No other hematologic or non-hematologic adverse effects occurred.

A total of eight patients completed the eight cycles of rituximab maintenance therapy. Two patients withdrew from the study due to relapse after receiving four and six courses

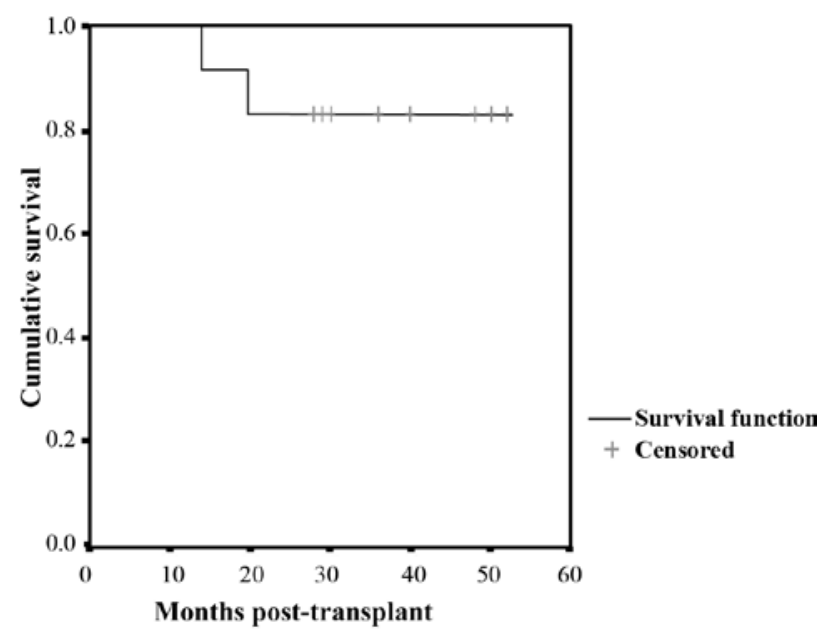

Figure 1. Progression-free survival in 12 patients with DLBCL who underwent auto-SCT combined with in vivo rituximab purging and post-transplant rituximab maintenance therapy. 
of rituximab maintenance. The remaining two patients, in whom the treatment interval was prolonged due to adverse effects, received four and seven courses of rituximab maintenance therapy. During maintenance therapy six months after undergoing auto-SCT, transient severe neutropenia (which lasted nine months) was observed in one patient. However, no signs of infection were noted, and the regimen was maintained. Herpes zoster was observed in one patient five months after undergoing transplantation; the patient recovered after receiving appropriate treatment. Repeated urinary tract infections and fungal vaginitis were observed in another patient six months after stem cell transplantation. However, the patient recovered two months after administration of antibacterial and anti-fungal therapy.

$H B V$ monitoring. HBV-related serologic markers (HBsAg, $\mathrm{HBs} \mathrm{Ab}, \mathrm{HBcAb}, \mathrm{HBeAg}$ and $\mathrm{HBeAb}$ ) were not detected in three patients before auto-SCT. Four patients were $\mathrm{HBsAb}$ positive, three were $\mathrm{HBsAb}$ - and $\mathrm{HBcAb}$-positive, and two were $\mathrm{HBcAb}$-positive. The five patients who were $\mathrm{HBcAb}$ positive received oral lamivudine. No changes in HBV-related markers were observed during follow-up. For the two patients who were only positive for $\mathrm{HBcAb}, \mathrm{HBV}-\mathrm{DNA}$ was also monitored and showed normal levels during follow-up.

Monitoring of immunoglobulin levels. Serum levels of immunoglobulins were monitored prior to stem cell transplantation and every three months during follow-up. The serum levels of immunoglobulins were normal in all 12 patients before autoSCT. After transplantation, reduced immunoglobulin levels were noted in two patients, six and 21 months after transplantation. Serum IgG and IgA levels were reduced by 50 and $30 \%$, respectively, in the first patient, accompanied by recurrent urinary tract infections and fungal vaginitis. Rituximab was withdrawn, and antibiotics and immunoglobulins were administered intravenously. The symptoms resolved two months later, and the patient's serum immunoglobulin levels recovered six months later. Subsequently, rituximab maintenance was reintroduced with no accompanying decrease in serum immunoglobulin levels. In the second patient, serum $\operatorname{IgM}$ and $\operatorname{IgA}$ levels were reduced by $>50 \% 21$ months after auto-SCT, and monoclonal immunoglobulin was not detected by immunofixation electrophoresis. Rituximab maintenance therapy was discontinued, and the patient was followed up for six months. Serum immunoglobulin levels partially recovered, but were lower than normal; however, no infection was observed in this patient.

\section{Discussion}

The introduction of rituximab has greatly improved treatment response, overall survival and disease-free survival in patients with DLBCL $(4,5)$. Nevertheless, tumor relapse is a frequent occurrence in all patient subsets, including younger patients with high-risk DLBCL. One approach to the treatment of relapsed DLBCL is the application of high-dose chemotherapy and auto-SCT $(14,15)$. Although these therapeutic regimens are effective at improving response to treatment, long-term and event-free survival have only partially improved. In this setting, residual disease and tumor contamination of trans- fused stem cells are the main causes for relapse of DLBCL. The presence of minimal residual disease (MRD) is a known poor prognostic indicator in DLBCL patients who undergo transplantation (16).

To this end, the eradication of tumor cells has been a treatment objective in therapeutic regimens involving transplantation. The use of rituximab for the in vivo purging of hematopoetic stem cell products has yielded encouraging results in disease eradication (up to $79 \%$ of MRD rendered undetectable after in vivo purging with rituximab), resulting in five-year OS and PFS of up to 78\% (16-19). An ongoing prospective study in patients with relapsed follicular lymphoma showed that the use of rituximab for in vivo graft purging and maintenance therapy following auto-STC resulted in undetectable MRD in a high proportion of patients (12). In this study, MRD was present in $56.5 \%$ of the patients (13/23) prior to treatment, but became undetectable in $77 \%$ of the patients after auto-SCT and in another two patients after subsequent rituximab maintenance therapy. Five-year OS and PFS were 78 and $59 \%$, respectively. Taken together, these results suggest that the use of rituximab for in vivo purging in combination with maintenance therapy is a feasible treatment option for patients with relapsing disease (11).

We hypothesized that rituximab purging and maintenance therapy in combination with auto-SCT is effective in DLBCL patients with $\mathrm{CR}$ after conventional induction treatment. Although our study was a single-center, prospective trial with the limitations of sample size and lack of a comparator group, our results provide further evidence supporting the use of rituximab in purging and maintenance therapy regimens.

The three-year and five-year OS rates in this study were $100 \%$, and the three-year and five-year PFS rates were $83 \%$ after rituximab maintenance therapy. Our results corroborate previously published findings using similar treatment regimens incorporating auto-SCT. Two prospective studies (LNH87 and LNH93) by the Groupe d'Etude des Lymphomes de l'Adulte (GELA) in 137 patients with DLBCL who received first-line transplantation showed five-year OS and disease-free survival rates of 76 and $68 \%$, respectively (20). Similar findings were obtained in an independent study in 112 previously untreated DLBCL patients who received a combination of rituximab and high-dose sequential chemotherapy alongside multiple autologous peripheral blood progenitor cell (PBPC) support (15). A total of $80 \%$ achieved CR, and the projected four-year OS and PFS were 76 and $73 \%$, respectively.

Although rituximab is a well-established component of induction therapy, concerns have been raised regarding the potential effects of rituximab-based induction therapy on the outcomes of re-treatment with rituximab. Further compounding the issue are conflicting results on the use of rituximab as maintenance therapy. Some studies demonstrated the benefits of rituximab in maintenance therapy, but the results are hampered by the small sample size (21). Other studies reported no significant differences in OS and PFS between DLBCL patients who received rituximab purging and maintenance therapy and those in the control group (22). The results from our study suggest that rituximab maintenance therapy following auto-STC is beneficial for DLBCL patients who received rituximabbased induction therapy. Three months after transplantation, 11 patients (91.7\%) achieved CR and one patient achieved CRu. 
After two cycles of rituximab maintenance therapy, CR was achieved in all 12 patients. A total of 10 patients achieved longterm CR and two experienced relapse 14 and 20 months after the end of rituximab maintenance therapy.

The safety findings in the present study did not differ from those previously reported (12), confirming the safety of rituximab in maintenance therapy. Treatment was delayed in only two patients due to rituximab toxicity. One adverse effect of potential concern is the activation of HBV replication, particularly in countries with a high incidence of HBV infection (such as China). The long duration of rituximab maintenance therapy may necessitate an increased frequency of anti-viral medication. In the present study, the activation of HBV replication was not observed upon treatment with rituximab. No patients withdrew from the study, and there were no delays in the start of therapy. Patients with active HBV replication were excluded, and lamivudine was administered to those who were positive for HBV-related antibodies (with the exception of HBsAb). Nevertheless, the feasibility of rituximab maintenance therapy in patients with signs of active HBV infection needs to be confirmed in larger patient cohorts.

Two patients exhibited markedly reduced levels of serum immunoglobulins. The patients recovered gradually following discontinuation of therapy, with one of these patients experiencing recurrent infections. Lim et al (23) and Nishio et al (24) reported that rituximab maintenance therapy following stem cell transplantation in NHL patients induced a decrease in serum immunoglobulin levels. Non-fatal infections were found in approximately 20-30\% of patients. In addition, Nishio et al (24) reported that the recovery of memory B cells in these patients was delayed, leading to a reduced expression of CD27, CD80 and CD40, and a corresponding decrease in the production of plasma cells. Taken together, these results highlight the importance of monitoring serum immunoglobulin levels during rituximab maintenance therapy.

There is currently much variability in the frequency and duration of rituximab use in maintenance therapy regimens. Treatment in the PRIMA and SAKK trials consisted of treatment cycles once every two or three months over two years (25). Other protocols define one treatment course as weekly rituximab treatment cycles over six consecutive weeks, and recommend one course of treatment every six months to a total of four courses. No conclusive evidence is currently available to support the clinical superiority of any treatment regimen. The results from our study suggest that a treatment cycle once every three months is an effective and practical alternative. Due to the long half-life of rituximab, effective plasma concentrations can be maintained with this regimen (26). Using this strategy, only eight treatment cycles are needed, thereby reducing treatment costs. The higher number of Chinese patients with potential HBV infections precludes the use of a once-weekly treatment regimen, which may activate HBV replication and increase the risk of liver deterioration. Nevertheless, this hypothesis needs to be tested in future clinical trials.

In conclusion, the results from our study suggest that OS and PFS in younger patients with high-risk DLBCL improved through rituximab purging and maintenance therapy combined with auto-SCT. These findings also confirm the safety and tolerability of rituximab in a clinical setting.

\section{References}

1. The Non-Hodgkin's Lymphoma Pathologic Classification Project. National Cancer Institute sponsored study of classifications of non-Hodgkin's lymphomas: summary and description of a working formulation for clinical usage. Cancer 49: 2112-2135, 1982.

2. The International Non-Hodgkin's Lymphoma Prognostic Factors Project. A predictive model for aggressive non-Hodgkin's lymphoma. N Engl J Med 329: 987-994, 1993.

3. Tilly $\mathrm{H}$ and Zelenetz A: Treatment of follicular lymphoma: current status. Leuk Lymphoma 49 (Suppl 1): S7-S17, 2008.

4. Coiffier B, Lepage E, Briere J, et al: CHOP chemotherapy plus rituximab compared with $\mathrm{CHOP}$ alone in elderly patients with diffuse large-B-cell lymphoma. N Engl J Med 346: 235-242, 2002.

5. Pfreundschuh M, Trumper L, Osterborg A, et al: CHOP-like chemotherapy plus rituximab versus CHOP-like chemotherapy alone in young patients with good-prognosis diffuse large-Bcell lymphoma: a randomised controlled trial by the MabThera International Trial (MInT) Group. Lancet Oncol 7: 379-391, 2006.

6. Sehn LH, Donaldson J, Chhanabhai M, et al: Introduction of combined CHOP plus rituximab therapy dramatically improved outcome of diffuse large B-cell lymphoma in British Columbia. J Clin Oncol 23: 5027-5033, 2005.

7. Thieblemont $\mathrm{C}$ and Gisselbrecht $\mathrm{C}$ : Second-line treatment paradigms for diffuse large B-cell lymphomas. Curr Oncol Rep 11: 386-393, 2009.

8. Cerny J, Trneny M, Slavickova A, et al: Rituximab based therapy followed by autologous stem cell transplantation leads to superior outcome and high rates of PCR negativity in patients with indolent B-cell lymphoproliferative disorders. Hematology 14: 187-197, 2009.

9. Philip T, Guglielmi C, Hagenbeek A, et al: Autologous bone marrow transplantation as compared with salvage chemotherapy in relapses of chemotherapy-sensitive non-Hodgkin's lymphoma. N Engl J Med 333: 1540-1545, 1995

10. Flohr T, Hess G, Kolbe K, et al: Rituximab in vivo purging is safe and effective in combination with CD34-positive selected autologous stem cell transplantation for salvage therapy in B-NHL. Bone Marrow Transplant 29: 769-775, 2002.

11. Magni M, Di NM, Devizzi L, et al: Successful in vivo purging of CD34-containing peripheral blood harvests in mantle cell and indolent lymphoma: evidence for a role of both chemotherapy and rituximab infusion. Blood 96: 864-869, 2000.

12. Hicks LK, Woods A, Buckstein R, et al: Rituximab purging and maintenance combined with auto-SCT: long-term molecular remissions and prolonged hypogammaglobulinemia in relapsed follicular lymphoma. Bone Marrow Transplant 43: 701-708, 2009.

13. Cheson BD, Horning SJ, Coiffier B, et al: Report of an international workshop to standardize response criteria for nonHodgkin's lymphomas. NCI Sponsored International Working Group. J Clin Oncol 17: 1244, 1999.

14. Haioun C, Lepage E, Gisselbrecht C, et al: Survival benefit of high-dose therapy in poor-risk aggressive non-Hodgkin's lymphoma: final analysis of the prospective LNH87-2 protocol - a Groupe d'Etude des Lymphomes de l'Adulte study. J Clin Oncol 18: 3025-3030, 2000.

15. Tarella C, Zanni M, Di NM, et al: Prolonged survival in poor-risk diffuse large B-cell lymphoma following front-line treatment with rituximab-supplemented, early-intensified chemotherapy with multiple autologous hematopoietic stem cell support: a multicenter study by GITIL (Gruppo Italiano Terapie Innovative nei Linfomi). Leukemia 21: 1802-1811, 2007.

16. Jacquy C, Soree A, Lambert F, et al: A quantitative study of peripheral blood stem cell contamination in diffuse large-cell non-Hodgkin's lymphoma: one-half of patients significantly mobilize malignant cells. Br J Haematol 110: 631-637, 2000.

17. Goldberg S, Pecora A, Jennis A, et al: Rituximab permits in vivo purging and collection of tumor-free stem cells prior to autologous transplantation for B-cell non-Hodgkin's lymphoma. Blood 94: $141 \mathrm{a}, 1999$.

18. Haioun C, Delrau-Larue M, Beaujean F, et al: Efficiency of in vivo purging with rituximab before autologous peripheral blood stem cell transplantation (PBSCT) in B-cell non-Hodgkin's lymphoma (NHL). Blood 96: 384a, 2000. 
19. Salles G, Moullet I, Charlot $\mathrm{C}$, et al: In vivo purging with rituximab before autologous peripheral blood progenitor cell (PBPC) transplantation in lymphoma patients. Blood 94: 141a, 1999.

20. Mounier N, Gisselbrecht C, Briere J, et al: All aggressive lymphoma subtypes do not share similar outcome after front-line autotransplantation: a matched-control analysis by the Groupe d'Etude des Lymphomes de l'Adulte (GELA). Ann Oncol 15 $1790-1797,2004$

21. Horwitz SM, Negrin RS, Blume KG, et al: Rituximab as adjuvant to high-dose therapy and autologous hematopoietic cell transplantation for aggressive non-Hodgkin lymphoma. Blood 103: 777-783, 2004

22. Habermann TM, Weller EA, Morrison VA, et al: RituximabCHOP versus CHOP alone or with maintenance rituximab in older patients with diffuse large B-cell lymphoma. J Clin Oncol 24: 3121-3127, 2006.

23. Lim SH, Zhang Y, Wang Z, et al: Maintenance rituximab after autologous stem cell transplant for high-risk B-cell lymphoma induces prolonged and severe hypogammaglobulinemia. Bone Marrow Transplant 35: 207-208, 2005.
24. Nishio M, Fujimoto K, Yamamoto S, et al: Delayed redistribution of CD27, CD40 and CD80 positive B cells and the impaired in vitro immunoglobulin production in patients with nonHodgkin lymphoma after rituximab treatment as an adjuvant to autologous stem cell transplantation. $\mathrm{Br} \mathrm{J}$ Haematol 137: 349-354, 2007.

25. Ghielmini M, Schmitz SF, Cogliatti S, et al: Effect of singleagent rituximab given at the standard schedule or as prolonged treatment in patients with mantle cell lymphoma: a study of the Swiss Group for Clinical Cancer Research (SAKK). J Clin Oncol 23: 705-711, 2005.

26. Cartron G, Blasco H, Paintaud G, Watier H and Le GC: Pharmacokinetics of rituximab and its clinical use: thought for the best use? Crit Rev Oncol Hematol 62: 43-52, 2007. 